DOI: https://doi.org/10.36548/jucct.2020.2.005

\title{
Computational Enhancements of Wearable Healthcare Devices on Pervasive Computing System
}

\author{
Dr. Subarna Shakya \\ Professor, \\ Department of Electronics and Computer Engineering, \\ Central Campus, Institute of Engineering, \\ Pulchowk,Tribhuvan University,Pulchowk, \\ Lalitpur Nepal. \\ Email: drss@ioe.edu.np
}

\begin{abstract}
The diverse user demands in the system supported with the internet of things are often managed efficiently, using the computing system that is pervasive. Pervasive computing system in an integration of heterogeneous distributed network and communication technologies and other referred as the ubiquitous computing. All though it handles the user requirement properly. The ingenuousness in the conveyance of the information, in the standard of handling and extending the heterogeneity assistance for the dispersed clients are still under construction in the as it is very challenging in the pervasive computing system. In order to provide proper and a steadfast communication for the users using an IOT based wearable health care device the paper introduces the new dispersed and elastic computing model (DECM). The developed system utilizes the recurrent-learning for the examining the allocation of resources according to the requirements as well as the allotment aspects. Based on the determined requirements of the resources, the pervasive computing system provide services to the user in the end with minimized delay and enhanced rate of communication for the health care wearable devices. The developed system emphasis also on managing the mobility, apart from allocation of resources and distribution for proper data conveyance over the wearable health care device. The working of the laid out system is determined by the experimental analysis. The constancy of the model developed is demonstrated utilizing the metrics such as the failure of request, time of response, managed and backlogged requests, bandwidth as well as storage used. The developed model heightens the number of request managed properly (handled) along with the bandwidth and storage and minimizes the failure in requests, backlogs and the time taken for response.
\end{abstract}

Keywords: Internet of Things, Wearable Devices, Pervasive Computing, Computational Improvements, Healthcare 
Journal of Ubiquitous Computing and Communication Technologies (UCCT) (2020)

Vol.02/ No. 02

Pages: 98-108

https://www.irojournals.com/jucct/

DOI: https://doi.org/10.36548/jucct.2020.2.005

\section{Introduction}

The recent developments of the applications that are capable of sensing and communicating ubiquitously has modernized and transformed the world into a "pervasive computing system environment (PCSE)". The device with the capacity to operate without wires, enriched with sensing units as well as processing and deciding capabilities are combined with the tangible world objects to create the proper service delivery for the internet of things. This sort of service are highly demanded for the commercial sectors, healthcare sectors, business and IT sectors, as well as in communicating and multimedia sectors. The user demands are satisfied by delivering a prompt service provisions, and by enhancing the "querying requests." PCSE combines a broad scope of "dispersed and local resources, the communication methodologies, the third party applications and the services." This PCSE follows a strategy that engages multiple layers that does the service-fetching from the outside paradigms like cloud computing and make sure that the required resources are available at the user edge i.e. the IOT based wearable heath care device (IOT-WHCD)

Integrating a broad range of devices ranging from sensors to intelligent equipment's is the key for accessing the network as well as the multitudes of resources. The consumers were allowed to have an undisturbed, ubiquitous right to use the resources all over the world by extending the communication via the devices of the end users. The equipment's enabled different variety of applications by connecting to the clients with the outside networks and the services via conveyance methodology that is adaptable.

The important requirements of the user in ubiquitous services provisioning is (i) focusing on the service, (ii) providing robust services satisfying the user requisitions. PCSE enables the right to use the devices that are heterogeneous as form of services. The service are provided via networks used for extending the communication by integrating the "communication interface of various service systems and the fundamental systems." PCSE is distributed system with the right to use the various computing paradigms in order to satisfy the demands of the users. Some of the service provided by the PCSE are the "simultaneous right to use for users, service configuration, computing questions, allotment of resources and distribution of resources". The data were dispersed across various health care sectors and flexibility in the conveyance was performed by combining various sensors clique supported by the internet of things. In order to accomplish a robust service for the edge users, the SDN, mobile-networks, medical sensor data centers, dispersed servers and the edge processing networks were incorporated.

In case of the large scale PCSE, extending a reliable and a flexible communication is essential but complicated and challenging. For the Iot based wearable health care device on the PCSE the paper has developed a new dispersed and elastic computing model (DECM). The developed system utilizes the

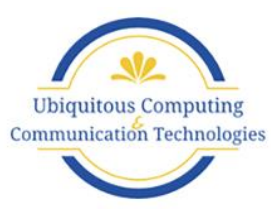


Journal of Ubiquitous Computing and Communication Technologies (UCCT) (2020)

Vol.02/ No. 02

Pages: 98-108

https://www.irojournals.com/jucct/

DOI: https://doi.org/10.36548/jucct.2020.2.005

recurrent-learning for the examining the allocation of resources according to the requirements as well as the allotment aspects. Based on the determined requirements of the resources, the pervasive computing system provide services to the user in the end with minimized delay and enhanced rate of communication for the health care wearable devices. The developed system emphasis also on managing the mobility, apart from allocation of resources and distribution for proper data conveyance over the wearable health care device.

- The laid out design improves the speed of computing the requests, by balancing the request flowing in the network. The balancing of the request is done utilizing the recurrent-learning. This brings down the response time and heightens the requests handled.

- The bandwidth rate is improved by optimizing the storage employing the recurrent learning.

- Experimental analysis of the laid out design and comparison of results with the existing methods.

The proposed methodology is formulated with the related works in section 2, proposed work in section 3, experimental analysis in section 4 and conclusion in section 5.

\section{Related Works}

Azariadi et al [1] has proposed a procedure to analyze the ECG and distinguish the heartbeat and further applied the procedure to a wearable health care device that does a constant $24 \times 7$ monitoring. Haghi et al [2] presents the review of WHCD both in the scientific papers and commercial efforts. Lomotey, et al [3] presented an "IOT architecture that for data streaming delivering a traceability of data route from the originating source to the Health data center, the method was devised to overcome the complexities in device data mapping and matching using the enhanced petri net service model."

Yang et al [4] "proposed a new method for ECG monitoring based on the IOT techniques, ECG data are collected utilizing the wearable monitoring node and are transmitted directly to the IOT cloud using Wi-Fi. Both the HTTP and he MQTTP are engaged in the IOT cloud in order to deliver visual and timely ECG data to users".

Hayek, et al [5] performed the "Smart wearable system for safety-related medical iot application: Case of epileptic patient working in industrial environment. "DE Silva, et al [6] "developed a remote care taking applications that could be integrated for patients. This system is utilized as a sensor integrated shoe and a

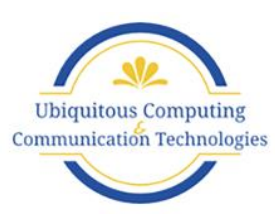


Journal of Ubiquitous Computing and Communication Technologies (UCCT) (2020)

Vol.02/ No. 02

Pages: 98-108

https://www.irojournals.com/jucct/

DOI: https://doi.org/10.36548/jucct.2020.2.005

waist belt. The data measured using the device is forwarded to the cloud and stored for further analysis and made available for the further researches.

Al-Makhadmeh, et al [7] utilizes the deep learning method and learns the previous analysis and does the prognosis of the disease related to the heart. The system utilized the "higher order Boltzmann deep belief neural networks". Baig et al [8] presents a "Systematic Review of Wearable Sensors and IoT-Based Monitoring Applications for Older Adults-a Focus on Ageing Population and Independent Living."

Sabban, Albert et al [9] does the "Compact Wearable Meta Materials Antennas for Energy Harvesting Systems, Medical and IOT Systems." Dey, Nilanjan et al [10] elaborated the challenges and the applications of the implantable and wearable medical devices. Balasubramaniam, et al [11] presented the "IoT-Based Noninvasive Wearable and Remote Intelligent Pervasive Healthcare Monitoring Systems for the Elderly People."

Greco, Luca et al [12] presented the "Trends in IoT based solutions for health care: moving AI to the Edge." Smys, S., et al [13] discussed the "Big Data Business Analytics as a Strategic Asset for Health Care Industry." Duraipandian et al [14] presented the "Cloud based Internet of Things for smart connected objects." Pandian, A. Pasumpon et al [15] performed the "Effective Fragmentation Minimization by Cloud Enabled Back up Storage." Joy Iong-Zong Chen et al [16] has discussed the. "Special Section on Innovative Engineering Solutions for Future Health Care Informatics." Raj, Jennifer S et al [17] has presented the. "Recurrent Neural Networks and Nonlinear Prediction in Support Vector Machines."

\section{Dispersed and Elastic Computing Model}

The flexibility and the elasticity are the inbuilt attributes of the PCSE, this enables the heterogeneous device operation providing an improved level of interoperability. The DECM proposed in the paper is utilized for delivering the service assistance for many users concurrently. The rate of conveyance in delivering the service is improved by computing the requests and the optimizing the storage using the recurrent-learning. The block diagram in figure. 1 presents the frame-work proposed.

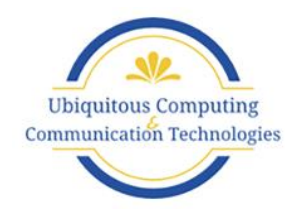


Vol.02/ No. 02

Pages: 98-108

https://www.irojournals.com/jucct/

DOI: https://doi.org/10.36548/jucct.2020.2.005

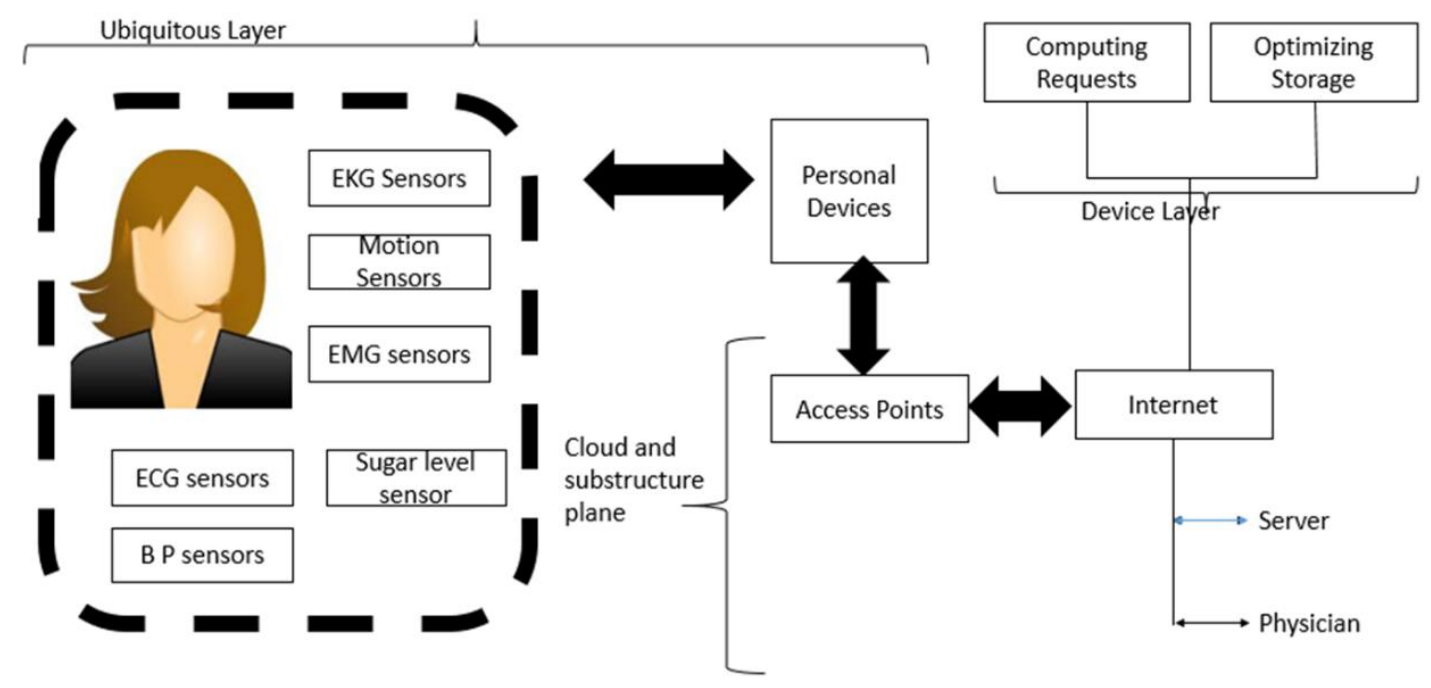

Figure.1 Block Diagram Proposed

The important planes that constitute the laid out design are the cloud, device, substructure, and the ubiquitous layer. The ubiquitous layer is enriched with various users, portable devices with IOT-WHCD that has requests for resources from the cloud plane. The plane accepts the applications that are mixed and the conveying methodology to examine the user requirements according to the standards of the information storage. The data analytics and the computing are also present in this plane. The device plane constitutes Control plane as in SDN and does the significant process the, computing of requests and the optimizing of storage to enhance the communication rate. The access points, gateways and the BS are equipped in the substructure plane that assist the heterogeneous communication incorporating a broad range of data conveyance technology that is based on the sensor associated with the internet of things. The significance of the substructure plane is its capability to cover broad scope of geographical area offering ubiquitous communication to the consumers. The cloud plane is a storehouse that stores the gathered information's to be used in the future. The committed cloud services handled the requisition of the client's about the information stored and takes the responsibility of computing and allocating the resources. Though the anytime, anywhere right to use is provided by the cloud for the authenticated clients it still work only as a third party.

To enhance the rate of conveyance the proposed design processes the request and optimizes the storage using the recurrent-learning. The processing the request enables one to arrange the request and deliver them to the cloud plane using the personal device either utilizes the Wi-Fi, or the Zig-Bee to upload the requests.

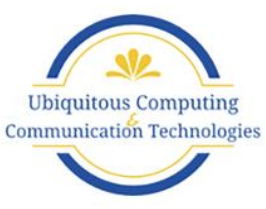


Journal of Ubiquitous Computing and Communication Technologies (UCCT) (2020)

Vol.02/ No. 02

Pages: 98-108

https://www.irojournals.com/jucct/

DOI: https://doi.org/10.36548/jucct.2020.2.005

This is minimizes the time of required in computing the requests and also the blocking possibilities and provides the seamless flow of data in the PCSE the request processing rate is estimated as in equation 1

$$
\text { Request processing rate }=\frac{\text { rate of requests } \mathrm{x} \text { number of devces }}{\text { maximum time limit }}
$$

Since the environment is pervasive the requests rate and the devices number keep on changing altering the processing rate of the requests, so the scalability function for the present rate of processing and the change processing rate are fixed equal to 1 , to minimize the processing time and process maximum of request from maximum devices. The scalability function is achieved using the two factors device connectivity probability and the rate of request arrival as in equation 2 and 3

$$
\text { Device Connectivity Probabilty }=\rho \text { devices } \frac{1}{\tau} \forall \text { devices } \geq \text { maximum connectivity }
$$

Where $\rho=$ normalization factor $\tau=$ exponent for connectivity the rate of arrival is shown in the equation 3

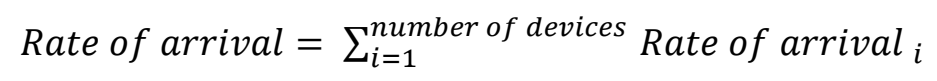

Based on the above equations the balancing rate for the requests flowing in and out of the network to minimize the delay in the delivery is achieved using the equation (4)

$$
\begin{aligned}
& \text { balancing rate } \\
& =\left\{\begin{array}{l}
\text { Request processing rate X maximum time taken } X \text { processing time, for request flowing in } \\
\frac{(1-\Delta) X \text { service time }}{\text { rate of arrival }},
\end{array}\right.
\end{aligned}
$$


Journal of Ubiquitous Computing and Communication Technologies (UCCT) (2020)

Vol.02/ No. 02

Pages: 98-108

https://www.irojournals.com/jucct/

DOI: https://doi.org/10.36548/jucct.2020.2.005

The inconsistencies in the conveyance are managed by engaging the recurrent-learning. The require outcome is achieved by managing the errors in the hidden layers, the learning process for the request processing and the storage optimization is shown in figure. 2(a) and 2(b)

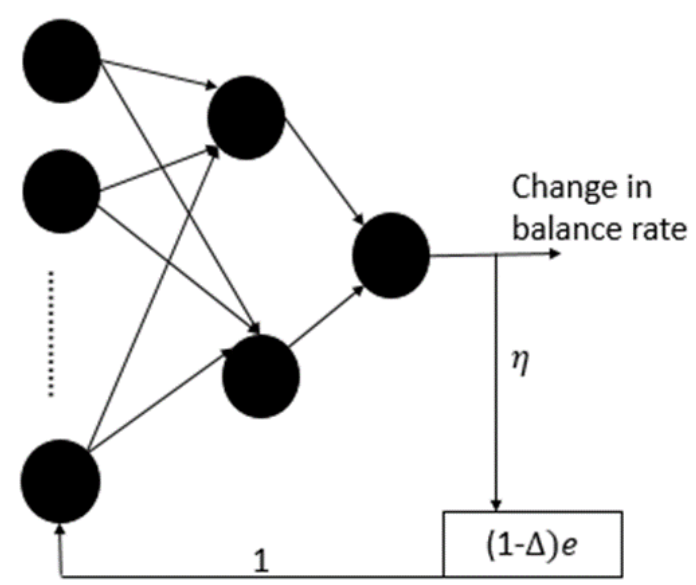

(a)

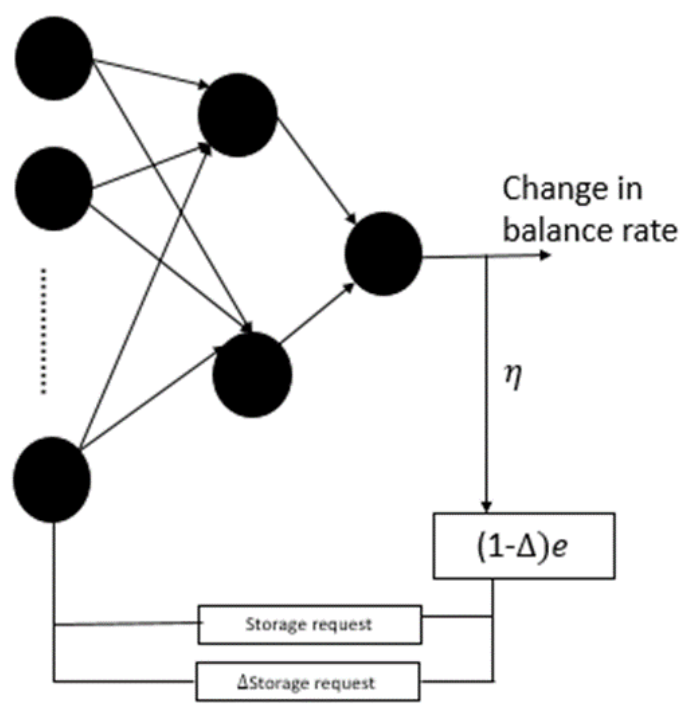

(b)

Figure. 2 Recurrent-Learning (a) Request Processing and (b) Storage

The prerequisite storage for the request is estimated using the learning process as shown in equation 5

$$
\text { Required stroage }=\frac{1}{\text { numbe rof requests }} \log \left(\frac{\frac{1}{l+1} \text { request } * \text { rate of arrival }}{1-\frac{\text { changein request rate }}{l-1}}\right)
$$

The computing of resources reduced the bottle necks in the conveyance of the requests and enhances the flow of request and the optimized storage assigns the storage for variety of requests with varying densities eluding the bottlenecks in the resource allocation process. 
Journal of Ubiquitous Computing and Communication Technologies (UCCT) (2020)

Vol.02/ No. 02

Pages: 98-108

https://www.irojournals.com/jucct/

DOI: https://doi.org/10.36548/jucct.2020.2.005

\section{Experimental Analysis}

The proposed mechanism is evaluated on a network simulator- 2 engaging more than 500 IOT based WHCD, the parameter used are listed in the table.1 below and the evaluation of the model consistency developed is demonstrated utilizing the metrics such as the failure of request, time of response, managed and backlogged requests, bandwidth as well as storage. The results obtained are compared with the existing methods to evince the robustness of the designed DECM.

\begin{tabular}{|c|c|}
\hline Parameters & Configurations \\
\hline WHCD & 500 \\
\hline Flow of Requests & 50 \\
\hline Pause Time & $5 \mathrm{~ms}$ \\
\hline Bandwidth & $2 \mathrm{Mbps}$ \\
\hline Maximum time & $20 \mathrm{~s}$ \\
\hline Storage size & 55 requests/second \\
\hline Number of requests & 600 requests/second \\
\hline
\end{tabular}

Table.1 Parameter and Configuration

The time taken for providing the response is presented is mapped in the figure. 3 , as the computing of the request reduces the backlogs and speeds up the process minimizing the delay and as well as minimizing the average response time required compared to the existing.

The balancing rate makes sure that the maximum number of requests are serviced in the with the specified service time minimizing the time taken for response. With the help of the optimization process over the storage, the requests are allotted with the proper resources in a linear mode taking into consideration the units of storage and changing the consecutive requests 
Journal of Ubiquitous Computing and Communication Technologies (UCCT) (2020)

Vol.02/ No. 02

Pages: 98-108

https://www.irojournals.com/jucct/

DOI: https://doi.org/10.36548/jucct.2020.2.005

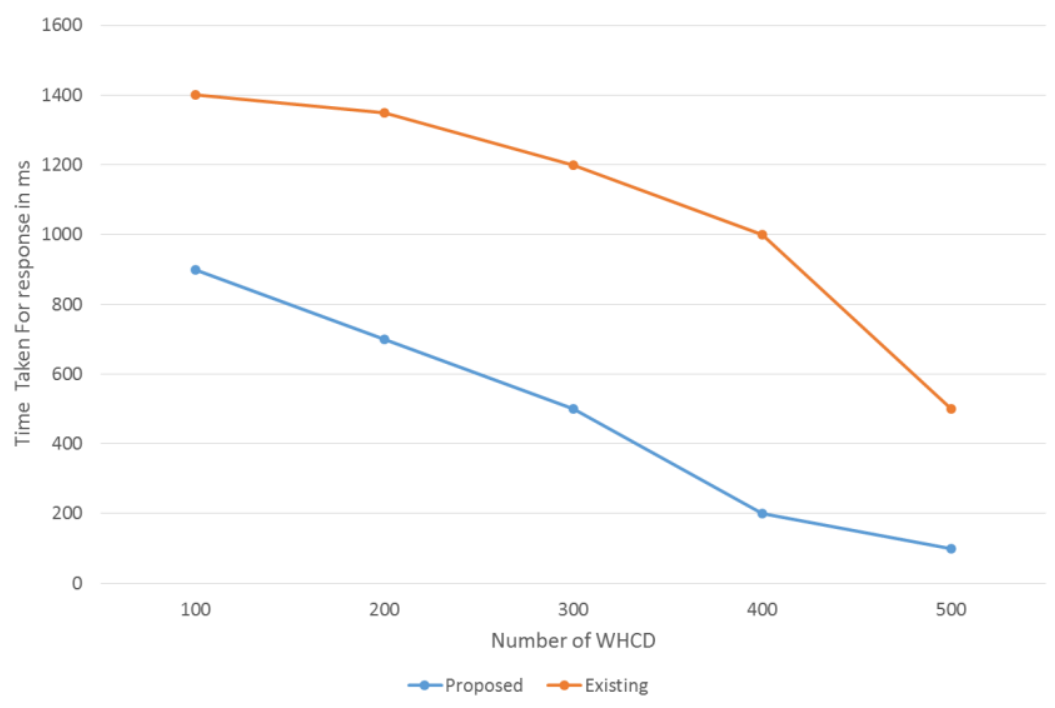

Figure. 3 Time Taken for Response

\begin{tabular}{|l|l|l|l|l|}
\hline \multirow{2}{*}{ Request Handled } & \multicolumn{2}{|c|}{ Existing } & \multicolumn{2}{c|}{ proposed } \\
\cline { 2 - 5 } & Failures & Backlogs & Failures & Backlogs \\
\hline 5000 & .30 & 405 & .12 & 100 \\
\hline 7000 & .35 & 500 & .14 & 125 \\
\hline 10000 & .39 & 900 & .15 & 200 \\
\hline 12000 & .45 & 850 & .12 & 234 \\
\hline 130000 & .50 & 890 & .13 & 225 \\
\hline
\end{tabular}

Table. 2 Request Handled, Backlogs, Failures

The RRL process engaged for minimizing the error in the requests received and for optimizing the storage enhances the resource allotment process, improving the dormantness in managing the requests and heightens the count id requests handled as well as computed by the system designed. The resource handled, the number of backlogs and the failures experienced in the existing and the proposed design are compared in the table. 2 
Journal of Ubiquitous Computing and Communication Technologies (UCCT) (2020)

Vol.02/ No. 02

Pages: 98-108

https://www.irojournals.com/jucct/

DOI: https://doi.org/10.36548/jucct.2020.2.005

\section{Conclusion}

DECM designed in the paper enhances the conveyance robustness, of the ubiquitous systems for the IOT based WHCD. The RRL process used computes the requests and optimizes the storage minimizing the bottle necks in the conveyance of the requests and enhancing the flow of request. Further the optimized storage assigns the storage for variety of requests with varying densities eluding the bottlenecks in the resource allocation process. The experiment observations demonstrates the constancy of the proposed designed against the existing system. The future scope of the paper is to develop the system supporting the edge network with supplementary enhancements to aid the simultaneous heterogeneous applications.

\section{References}

[1] Azariadi, Dimitra, Vasileios Tsoutsouras, Sotirios Xydis, and Dimitrios Soudris. "ECG signal analysis and arrhythmia detection on IoT wearable medical devices." In 2016 5th International conference on modern circuits and systems technologies (MOCAST), pp. 1-4. IEEE, 2016.

[2] Haghi, Mostafa, Kerstin Thurow, and Regina Stoll. "Wearable devices in medical internet of things: scientific research and commercially available devices." Healthcare informatics research 23, no. 1 (2017): 4-15.

[3] Lomotey, Richard K., Joseph Pry, and Sumanth Sriramoju. "Wearable IoT data stream traceability in a distributed health information system." Pervasive and Mobile Computing 40 (2017): 692-707.

[4] Yang, Zhe, Qihao Zhou, Lei Lei, Kan Zheng, and Wei Xiang. "An IoT-cloud based wearable ECG monitoring system for smart healthcare." Journal of medical systems 40, no. 12 (2016): 286.

[5] Hayek, Ali, Samer Telawi, Josef Börcsök, Roy Abi Zeid Daou, and Nashaat Halabi. "Smart wearable system for safety-related medical iot application: Case of epileptic patient working in industrial environment." Health and Technology 10, no. 1 (2020): 363-372.

[6] De Silva, AHT Eranga, WH Peshan Sampath, NH Lakshitha Sameera, YW Ranjith Amarasinghe, and Atsushi Mitani. "Development of a wearable tele-monitoring system with IoT for bio-medical applications." In 2016 IEEE 5th global conference on consumer Electronics, pp. 1-2. IEEE, 2016.

[7] Al-Makhadmeh, Zafer, and Amr Tolba. "Utilizing IoT wearable medical device for heart disease prediction using higher order Boltzmann model: A classification approach." Measurement 147 (2019): 106815.

[8] Baig, Mirza Mansoor, Shereen Afifi, Hamid GholamHosseini, and Farhaan Mirza. "A Systematic Review of Wearable Sensors and IoT-Based Monitoring Applications for Older

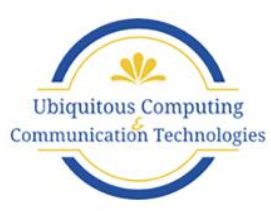


Journal of Ubiquitous Computing and Communication Technologies (UCCT) (2020)

Vol.02/ No. 02

Pages: 98-108

https://www.irojournals.com/jucct/

DOI: https://doi.org/10.36548/jucct.2020.2.005

Adults-a Focus on Ageing Population and Independent Living." Journal of medical systems 43, no. 8 (2019): 233.

[9] Sabban, Albert. "Compact Wearable Meta Materials Antennas for Energy Harvesting Systems, Medical and IOT Systems." Electronics 8, no. 11 (2019): 1340.

[10] Dey, Nilanjan, Amira S. Ashour, Simon James Fong, and Chintan Bhatt, eds. Wearable and Implantable Medical Devices: Applications and Challenges. Academic Press, 2019.

[11] Balasubramaniam, Sathiyabhama, and Rajeswari Kurubarahalli Chinnasamy. "IoT-Based Noninvasive Wearable and Remote Intelligent Pervasive Healthcare Monitoring Systems for the Elderly People." Intelligent Pervasive Computing Systems for Smarter Healthcare (2019): 141-158.

[12] Greco, Luca, Gennaro Percannella, Pierluigi Ritrovato, Francesco Tortorella, and Mario Vento. "Trends in IoT based solutions for health care: moving AI to the Edge." Pattern Recognition Letters (2020).

[13] Smys, S., and C. V. Joe. "Big Data Business Analytics as a Strategic Asset for Health Care Industry." Journal of ISMAC 1, no. 02 (2019): 92-100.

[14] Duraipandian, M., and Mr R. Vinothkanna. "Cloud based Internet of Things for smart connected objects." Journal of ISMAC 1, no. 02 (2019): 111-119.

[15] Pandian, A. Pasumpon, and S. Smys. "Effective Fragmentation Minimization by Cloud Enabled Back Up Storage." Journal of Ubiquitous Computing and Communication Technologies (UCCT) 2, no. 01 (2020): 1-9.

[16] Smys, S., and Joy Iong-Zong Chen. "Special Section on Innovative Engineering Solutions for Future Health Care Informatics." Journal of Medical Imaging and Health Informatics 6, no. 7 (2016): 1570-1571.

[17] Raj, Jennifer S., and J. Vijitha Ananthi. "Recurrent Neural Networks and Nonlinear Prediction in Support Vector Machines." Journal of Soft Computing Paradigm (JSCP) 1, no. 01 (2019): 33-40.

\section{Authors Biography}

Dr. Prof. Subarna Shakya is currently a Professorof Computer Engineering, Department of Electronics and Computer Engineering, Central Campus, Institute of Engineering, Pulchowk, Tribhuvan University, Coordinator (IOE), LEADER Project (Links in Europe and Asia for engineering,education, Enterprise and Research exchanges), ERASMUS MUNDUS. She received MSc and PhD degrees in Computer Engineering from the Lviv Polytechnic National University, Ukraine, 1996 and 2000 respectively. Her research area includes E-Government system, Computer Systems \& Simulation,Distributed \& Cloud computing, Software Engineering \& Information System, Computer Architecture, Information Security for E-Government, Multimedia system

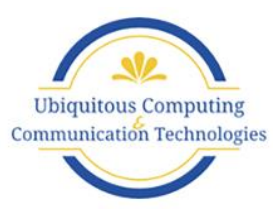

